\title{
Biodisponibilidade de minerais em refeições vegetarianas e onívoras servidas em restaurante universitário
}

\author{
Mineral bioavailability in vegetarian and omnivorous \\ meals served in a university restaurant
}

\author{
Egle Machado de Almeida SIQUEIRA \\ Juliana Frossard Ribeiro MENDES² \\ Sandra Fernandes ARRUDA ${ }^{1}$
}

\section{R E S U M O}

\section{Objetivo}

Avaliar a biodisponibilidade de cálcio, ferro e zinco nas dietas vegetarianas e onívoras, servidas no Restaurante Universitário da Universidade de Brasília.

\section{Métodos}

Foram coletadas três amostras de cada refeição, durante 5 dias, para determinação do conteúdo de fitato. O conteúdo em cálcio ferro e zinco foi estimado a partir de tabelas de composição de alimentos. A razão molar entre o ácido fítico e o mineral dietéticos foi utilizada como critério para avaliar a biodisponibilidade.

\section{Resultados}

As concentrações médias de ácido fítico, cálcio e ferro foram significantemente maiores na dieta vegetariana $(p=0,0002 ; p=0,0015$ e $p=0,0227$, respectivamente), enquanto a concentração de Zinco foi maior na dieta onívora $(p=0,0205)$. Ambas as dietas apresentaram razões molares ácido fítico: cálcio e ácido fítico: ferro inferiores aos valores críticos (1,56 e 14, respectivamente), sugerindo que o fitato não deve comprometer a biodisponibilidade do cálcio ou do ferro nessas refeições. A razão molar ácido fítico: zinco, na dieta vegetariana, foi de 9,3 e na onívora de 4,9, caracterizando-as como refeições de média e alta biodisponibilidade para o zinco, respectivamente. O feijão e a soja eram os componentes dietéticos mais ricos em fitato nas duas refeições.

\section{Conclusão}

Devido aos teores de cálcio e ferro nas duas refeições, a presença de fitato não deve representar risco de deficiência desses minerais para indivíduos que se alimentam diariamente no Restaurante Universitário da

\footnotetext{
1 Universidade de Brasília, Departamento de Biologia Celular, Laboratório de Biofísica. Asa Norte, Campus Universitário Darcy Ribeiro, ICC Sul, Módulo 3, 70910-900, Brasília, DF, Brasil. Correspondência para/Correspondence to: E.M.A. SIQUEIRA. E-mail: <eglemasi@gmail.com>.

2 Universidade de Brasília, Departamento de Nutrição. Brasília, DF, Brasil.
} 
Universidade de Brasília. Entretanto, devido ao baixo teor de zinco nas refeições, a presença do fitato pode representar um risco de deficiência desse mineral, principalmente, para homens vegetarianos.

Termos de indexação: vegetarianos; onívoros; biodisponibilidade; fitato; minerais; feijão.

\section{A B S T R A C T}

\section{Objective}

To assess the bioavailability of calcium, iron and zinc in vegetarian and omnivorous meals served in the university restaurant of the Universidade de Brasilia.

\section{Methods}

Three samples of each meal were collected during 5 days to determine the concentration of phytate. Calcium, iron and zinc contents were estimated by using food composition tables. The molar ratio between phytic acid and the dietary mineral was used as a criterion to evaluate mineral bioavailability.

\section{Results}

The mean concentrations of phytate, calcium and iron were significantly higher in the vegetarian meal ( $p=0.0002 ; p=0.0015$ e $p=0.0227$, respectively), while zinc concentration was higher in the omnivorous meal $(p=0.0205)$. Both meals presented molar ratios of phytate to calcium and phytate to iron below the critical values (1.56 and 14, respectively) suggesting that phytate in these meals should not impair calcium and iron bioavailability. The molar ratio of phytic acid to zinc in the vegetarian meal was 9.4, while in the omnivorous meal it was 4.3, characterizing them as medium and high bioavailability meals for zinc, respectively. Beans and soy were the highest sources of phytate in both meals.

\section{Conclusion}

Due to the calcium and iron contents in both meals served in the university restaurant of the Universidade de Brasilia, phytate content should not induce calcium and iron deficiency in individuals that consume these meals daily. However, given the low zinc content in the meals, phytate content may represent a risk for the development of zinc deficiency, especially in vegetarian men.

Indexing terms: vegetarian; omnivorous; biological availability; phytic acid; minerals; bean.

\section{N T R O D U Ç Ã O}

Nos últimos anos, as dietas vegetarianas vêm se tornando cada vez mais populares em vários países, por razões de saúde, filosóficas, ecológicas ou religiosas ${ }^{1,2}$. Um crescente número de evidências científicas tem indicado que as dietas vegetarianas oferecem vantagens significativas em relação às dietas onívoras, principalmente, devido ao reduzido teor de gorduras saturadas, de colesterol, de proteína animal, além de apresentarem maior conteúdo em fibras, carboidratos complexos e antioxidantes, que exercem impacto positivo na prevenção e no controle de doenças crônicas não transmissíveis ${ }^{2-4}$. Porém, as dietas vegetarianas podem resultar na deficiência de vitamina $B_{12}$, vitamina $B_{2}$, vitamina $D$, cálcio, ferro e zinco ${ }^{1,3,5}$. Dietas vegetarianas contêm grandes quantidades de fibras, fitato e oxalato, compostos capazes de quelar minerais, reduzindo a absorção destes no intestino ${ }^{6}$. $O$ ácido fítico (AF), abundante, principalmente, nos cereais integrais, no feijão e na soja, possui efeito quelante sobre íons mono e bivalentes ${ }^{7,8}$, formando complexos insolúveis, que resultam na redução da biodisponibilidade de minerais e de proteínas da dieta, o que lhe conferiu o status de antinutriente. Por outro lado, estudos mais recentes têm demonstrado o papel do fitato no combate à carcinogênese, às doenças cardiovasculares e na inibição da produção de radicais livres ${ }^{8-10}$.

A biodisponibilidade de minerais tem sido avaliada por meio das razões molares entre o fitato e os minerais da dieta. Saha et al. ${ }^{11}$ demonstraram 
que uma dieta com razão molar AF: Cálcio (Ca) igual a 1,56 apresentou comprometimento na absorção do íon Ca. Dietas com razão molar AF:Ferro (Fe) $>14$ comprometem a biodisponibilidade desses minerais em animais e humanos ${ }^{12,10}$. Segundo a Organização Mundial de Saúde (OMS), dietas com razão molar AF:Zn acima de 15 apresentam baixa biodisponibilidade de Zn (10\% a $15 \%)$, entre 5 e 15 apresentam média biodisponibilidade de Zn (30\% a 35\%) e, abaixo de 5 apresentam alta biodisponibilidade ${ }^{13}$. O fitato no organismo apresenta efeitos diversos, podendo ser benéfico ou prejudicial à saúde, dependendo, principalmente, da concentração dos outros nutrientes na dieta do indivíduo. O objetivo deste estudo foi avaliar a biodisponibilidade dos minerais nas refeições onívora e vegetariana, servidas no Restaurante Universitário da Universidade de Brasília (RU), tendo como parâmetro as razões molares entre esses minerais e o fitato.

\section{MÉTODOS}

Amostras de pratos (refeições onívoras e vegetarianas), servidas no almoço, foram coletadas durante cinco dias consecutivos, no RU, em triplicata, totalizando 30 refeições (15 onívoras e 15 vegetarianas). Os pratos foram montados por um dos pesquisadores, simulando as variações de um mesmo indivíduo. Os alimentos presentes em cada refeição foram pesados separadamente, para posterior estimativa da composição em minerais. O cardápio de ambas as refeições era composto, basicamente, por arroz, feijão, salada (vegetal A e B), sendo a carne, presente na refeição onívora, substituída por preparações à base de soja ou glúten na refeição vegetariana.

Os alimentos contidos nos pratos foram homogeneizados para determinação do teor de fitato, segundo Latta \& Eskin ${ }^{14}$. Para a extração foi dissolvido $1 \mathrm{~g}$ de amostra em $20 \mathrm{~mL}$ de $\mathrm{HCl}$ 2,4M, deixado sob agitação constante por uma hora, à temperatura ambiente, centrifugado por 10 minutos a $13.000 \mathrm{rpm}$, sendo o sobrenadante diluído com água destilada (1:10). Em seguida,
$10 \mathrm{~mL}$ da amostra diluída foram aplicados em uma coluna aberta $(15 \times 1 \mathrm{~cm}, 200-400$ mesh, AG1-X8 - fase estacionária), previamente equilibrada com $25 \mathrm{~mL}$ de $\mathrm{NaCl}$ 0,7 M. O fósforo inorgânico livre foi, então, eluído com $15 \mathrm{~mL}$ de $\mathrm{NaCl} 0,1 \mathrm{M}$, seguido da eluição do fitato com $16 \mathrm{~mL}$ de $\mathrm{NaCl}$ 0,7 M. Frações de $2 \mathrm{~mL}$ foram coletadas e submetidas à análise colorimétrica para detecção do fitato [em $900 \mu \mathrm{L}$ da fração foi adicionado $300 \mu \mathrm{L}$ de reagente Wade (cloreto de ferro 0,03\% + ácido sulfosalicílico 0,3\%), centrifugação por 3 minutos a 13.000rpm, sendo a absorbância lida a 500nm. A concentração de fitato nas amostras foi determinada utilizando uma curva padrão, que variava de 5 a $45 \mu \mathrm{g} / \mathrm{mL}$ de ácido fítico da marca SIGMA ${ }^{\circledast}(y=0,0091 \mathrm{x}+$ 0,0219; $\left.R^{2}=0,99\right)$.

As concentrações de $\mathrm{Ca}$, Fe e $\mathrm{Zn}$ das refeições foram estimadas a partir de tabelas de composição de alimentos ${ }^{15,16}$.

Para a biodisponibilidade do Ca, Fe e Zn: foi utilizada a razão molar ácido fítico versus elemento (AF: Ca; AF: Fe e AF: Zn), para avaliar a biodisponibilidade desses minerais nas refeições, tendo como critério o valor crítico, descrito na literatura para essas razões ${ }^{11-13}$.

Para verificar a normalidade das distribuições, a densidade energética e os teores de minerais e fitato dietéticos, foi utilizado o teste de Kolmogorov-Smirnov (programa computacional SPSS). A igualdade entre as médias desses valores, obtida nas duas dietas, onívoras e vegetarianas, foi verificada pelo teste Anova fator único ${ }^{17}$. Nas comparações entre os percentuais das Dietary References Intakes (DRIs) para as duas dietas e o sexo, foi empregada análise de variância fator múltiplo (Anova com correção de Bonferroni - programa Excel). O nível de significância estabelecido para todas as comparações foi de $p<0,05$.

\section{RESULTADOS}

A refeição vegetariana apresentou densidade energética e concentrações de AF, Ca e Fe 
maiores que a refeição onívora $(p=0,025$; $p=0,0002 ; p=0,0015$ e $p=0,0227$, respectivamente), enquanto que a refeição onívora forneceu maior concentração de $\mathrm{Zn}$ em relação à vegetariana $(p=0,0205)$. As preparações à base de soja (refogada ou recheada com queijo) foram as que mais contribuíram para o maior valor energético oferecido pelas refeições vegetarianas (Tabela 1).

Uma avaliação da representatividade dessas refeições em relação às Dietary References Intakes (DRIs) ${ }^{18,19}$ para homens e mulheres entre 19 e 30 anos, mostra que as refeições vegeta-

Tabela 1. Teor de ácido fítico (AF) e dos minerais Ca, Fe e Zn das refeições onívora e vegetariana, oferecidas pelo Restaurante Universitário da Universidade de Brasília. Brasília, DF, 2001.

\begin{tabular}{|c|c|c|c|c|c|c|c|c|c|c|}
\hline \multirow{3}{*}{ Refeições ( $n=30$ ) } & \multicolumn{10}{|c|}{$(100 \mathrm{~g})$} \\
\hline & \multicolumn{2}{|c|}{ Energia (Kcal) } & \multicolumn{2}{|c|}{$\mathrm{AF}(\mathrm{Mg})$} & \multicolumn{2}{|c|}{$\mathrm{Ca}(\mathrm{Mg})$} & \multicolumn{2}{|c|}{$\mathrm{Fe}(\mathrm{Mg})$} & \multicolumn{2}{|c|}{$\mathrm{Zn}(\mathrm{Mg})$} \\
\hline & $\mathrm{M}$ & DP & M & DP & $\mathrm{M}$ & DP & $\mathrm{M}$ & $\mathrm{DP}$ & $\mathrm{M}$ & DP \\
\hline Onívora $(n=15)$ & 549,0 & $107,8^{b}$ & 44,0 & $14,1^{\text {a }}$ & 29,96 & $4,17^{a}$ & 1,54 & $0,26^{\mathbf{b}}$ & 1,03 & $0,25^{a}$ \\
\hline Vegetariana $(n=15)$ & 646,7 & $118,6^{a}$ & 78,3 & $22,7^{\mathbf{b}}$ & 55,41 & $27,80^{\mathbf{b}}$ & 1,87 & $0,46^{\mathrm{a}}$ & 0,84 & $0,14^{\mathrm{b}}$ \\
\hline
\end{tabular}

Os valores com letras diferentes, dentro da coluna, apresentaram diferenças significantes $(p<0,05)$. M: média; DP: desvio-padrão.

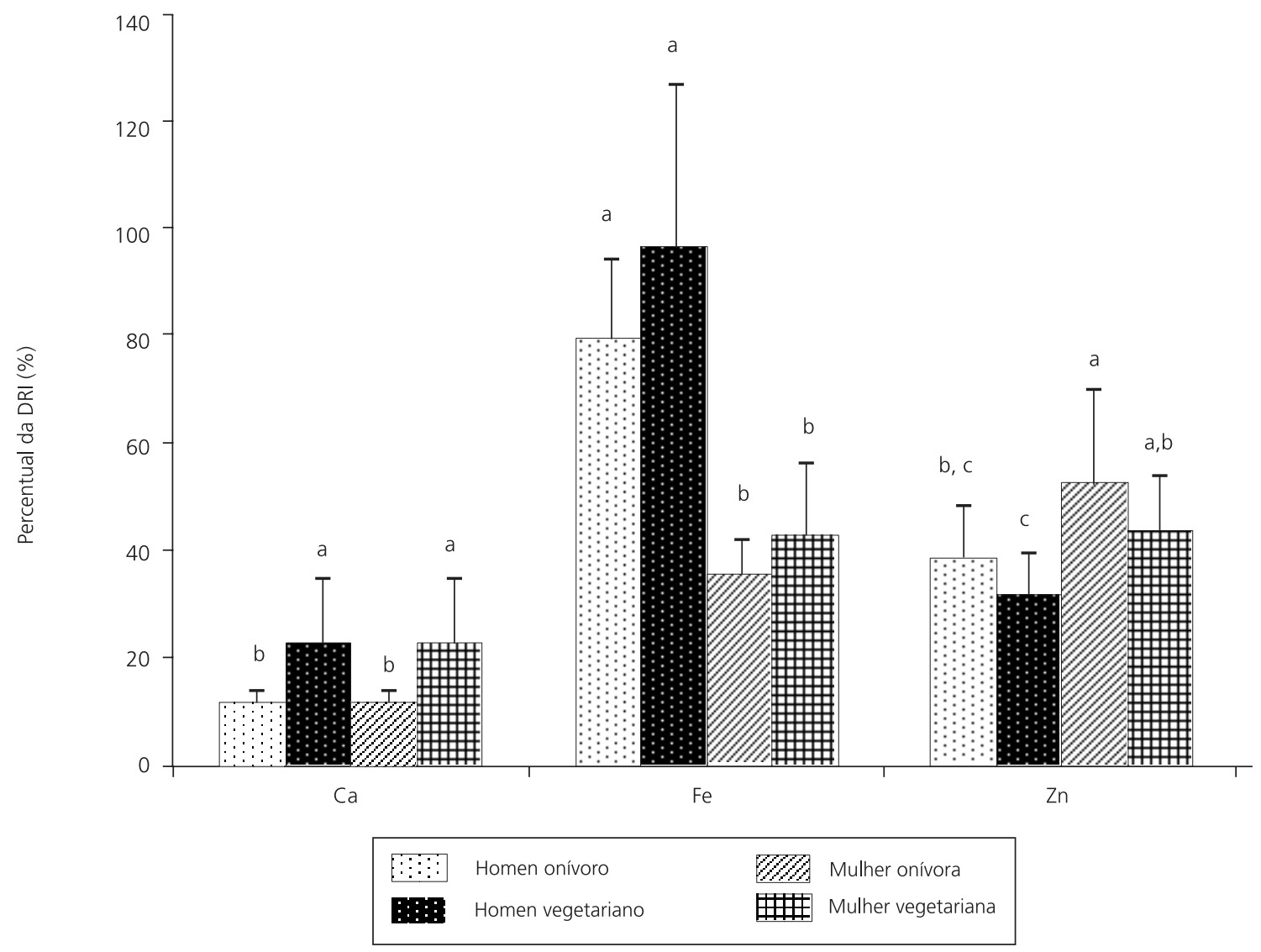

Figura 1. Porcentagem de contribuição das refeições onívora e vegetariana em relação às DRI de Ca, Fe e Zn, para indivíduos entre 19 e $30 \operatorname{anos}^{18,19}$. Brasília, DF, 2001.

Letras diferentes entre colunas representam diferença significante para $p<0,05$. 
rianas e onívoras contribuem, em média, com mais de $30 \%$ dos valores da Recommended Dietary Allowances (RDA) para Fe e Zn, sendo que o ferro, nas duas refeições, corresponde a $75 \%$ do valor de referência para homens. Por outro lado, a ingestão de Ca na refeição vegetariana não atinge $25 \%$ da Adequate Intake (Al) para o Ca e, na refeição onívora, esse valor reduz-se significantemente $(p=0,0112)$ para cerca de $12 \%$, em ambos os sexos (Figura 1).

Neste estudo, as razões molares entre o ácido fítico e os minerais, encontradas nas duas refeições, foram inferiores aos respectivos valores críticos $^{11-13}$. A refeição onívora apresentou razões molares AF: Fe e AF: Zn inferiores às da refeição vegetariana, não havendo diferença significante entre as razões molares AF: Ca das duas refeições (Tabela 2).

\section{I S C U S S Ã O}

De acordo com as recomendações de necessidade energética média diária da Organização Mundial de Saúde ${ }^{20}$, para indivíduos entre 18 e 30 anos, as refeições onívoras contribuiriam com $23 \%$ e $28 \%$ das recomendações, para homens e mulheres entre 70 e $60 \mathrm{~kg}$, respectivamente, enquanto as refeições vegetarianas com, aproximadamente, $27 \%$ e $33 \%$ das recomendações.

A alta concentração de fitato observada na refeição vegetariana deveu-se, principalmente, à presença de soja, em adição ao feijão, igualmente presente na refeição onívora. Esse fato, mais a presença esporádica de queijo, foram responsáveis pelo aumento significativo de cálcio na refeição vegetariana, em relação à refeição onívora. A maior ingestão de $\mathrm{Ca}$ em indivíduos ou populações lactoovovegetarianas, em relação a não vegetarianas, tem sido amplamente descrita na literatura, no entanto, indivíduos vegans (vegetarianos estritos) obtêm suprimento limitado de $\mathrm{Ca}$, devido à exclusão de alimentos fonte , como o leite e os derivados 6,21 .

Neste estudo, apesar da maior concentração de fitato na refeição vegetariana, a razão molar AF: Ca desse grupo não foi diferente da do grupo onívoro, devido, principalmente, à presença de queijo e soja, fontes de Ca na refeição vegetariana. Considerando que ambas as razões molares AF:Ca, encontradas nas duas refeições, apresentaram valores bem inferiores ao valor crítico, as refeições onívoras e vegetarianas servidas no RU não devem apresentar risco de comprometimento da biodisponibilidade do Ca devido à presença do ácido fítico. Estes resultados estão de acordo com a literatura, uma vez que os trabalhos publicados têm demonstrado que não há diferenças significativas na densidade óssea de mulheres pós-menopausa onívoras e lactoovovegetarianas, sendo aceito apenas um maior risco de osteoporose e comprometimento do crescimento entre os vegans ${ }^{22}$.

O ferro presente nos alimentos de origem vegetal (ferro inorgânico) é menos biodisponível que o ferro hêmico, encontrado nos alimentos de origem animal22, o que sugeriria um possível comprometimento do estado nutricional em ferro nos indivíduos vegetarianos. No entanto, os estudos têm demonstrado resultados contraditórios

Tabela 2. Razões molares médias entre AF e os minerais Ca, Fe e Zn das refeições onívoras e vegetariana analisadas, servidas pelo restaurante universitário de Brasília, DF, 2001.

\begin{tabular}{|c|c|c|c|c|c|c|}
\hline \multirow{2}{*}{ Refeição } & \multicolumn{2}{|c|}{ AF: $C a^{11}$} & \multicolumn{2}{|c|}{$A F: F e^{12}$} & \multicolumn{2}{|c|}{$\mathrm{AF}: \mathrm{Zn}^{13}$} \\
\hline & $\mathrm{M}$ & DP & M & DP & $M$ & $\mathrm{DP}$ \\
\hline Onívora & 0,09 & $0,02^{a}$ & 2,47 & $0,85^{\mathbf{b}}$ & 4,86 & $2,06^{b}$ \\
\hline Vegetariana & 0,09 & $0,04^{a}$ & 3,77 & $1,40^{\mathrm{a}}$ & 9,31 & $2,71^{a}$ \\
\hline Valores críticos & \multicolumn{2}{|c|}{$>1,56^{1}$} & \multicolumn{2}{|c|}{$>14^{2}$} & \multicolumn{2}{|c|}{$>15^{3}$} \\
\hline
\end{tabular}

Os valores com letras diferentes dentro da coluna apresentaram diferenças significantes $(p<0,025)$. M: média; DP: desvio-padrão. 
quanto à influência da forma em que o ferro se apresenta no alimento sobre o estado nutricional em ferro nos vegetarianos. Wilson \& Ball ${ }^{3}$ constataram que, mesmo entre vegetarianos que tinham maior ingestão de $\mathrm{Fe}$, comparados a onívoros, os níveis de ferritina sérica e concentração de hemoglobina eram significativamente menores. Porém, Hunt \& Roughead ${ }^{23}$ não observaram alteração nos níveis séricos de hemoglobina, saturação de transferrina, protoporfirina eritrocitária e ferritina, em indivíduos que consumiram dietas onívoras e lactoovovegetarianas, durante oito semanas. Nesse estudo foi observada uma redução da excreção fecal da ferritina entre os indivíduos com a dieta vegetariana, sugerindo uma adaptação fisiológica. Os autores acreditam que um aumento no tempo de acompanhamento dos indivíduos possa alterar esses resultados.

Haddad et al..$^{24}$ demonstraram que os vegetarianos possuem menor concentração de ferritina e hemoglobina, em relação aos onívoros, porém, não são deficientes em ferro, pois apresentam níveis de hemoglobina e ferritina sérica acima dos valores limítrofes. No presente estudo, embora a razão $A F$ :Fe tenha sido significantemente maior na refeição vegetariana, esse valor não atingiu $30,0 \%$ do valor crítico. Este resultado sugere uma alta biodisponibilidade para o Fe em ambas as refeições. Contudo, considerando que na refeição vegetariana o Fe inorgânico seja a forma predominante e que na refeição onívora seja o ferro hêmico, espera-se que a biodisponibilidade do Fe na refeição vegetariana seja menor que na onívora. Este fato não deve comprometer o suprimento de ferro da refeição vegetariana, pelo menos para indivíduos do sexo masculino, uma vez que essa refeição, sozinha, oferece cerca de $95,5 \%$ do valor de referência diário para homens entre 19 e 30 anos. Para mulheres, isso pode representar um risco para deficiência, não devido à presença de fitato, mas à forma do Fe encontrada na refeição vegetariana.

A biodisponibilidade, tanto do cálcio e do ferro, quanto a do zinco é inversamente proporcional à sua razão molar AF:Elemento. Segundo estimativas da World Health Organization (WHO), dietas com uma razão molar AF:Zn acima de 15 apresentam biodisponibilidade de zinco relativamente pobre (10\% a $15 \%)$, aquelas com razão molar entre 5 e 15, apresentam média biodisponibilidade (30\% a 35\%), e aquelas com razão molar abaixo de 5 , alta biodisponibilidade de zinco (45\% a 55\%) $)^{25}$. Dados limitados sobre absorção de zinco da dieta total em humanos sugerem uma influência do fitato mais moderada, resultando em $26 \%$ a $33 \%$ de absorção desse mineral em dietas com razões molares de 14 e 5 , respectivamente ${ }^{26}$, e $29 \%$ de absorção em dietas com razão molar de $7^{27}$. Estudo realizado com mulheres caucasianas australianas revelou que, embora o consumo médio de zinco no grupo de vegetarianas fosse significantemente menor que nas onívoras, as médias de concentrações de zinco sérico eram similares nos dois grupos ${ }^{1}$. Alguns estudos têm sugerido um aumento da absorção do Zn em indivíduos com baixa ingestão ou deficiência desse mineral ${ }^{1,6}$.

Freeland-Graves ${ }^{6}$ observou, em indivíduos não vegetarianos, submetidos a uma dieta lactoovovegetariana, por 22 dias, um aumento significante na resposta ao teste de tolerância ao Zn plasmático, sugerindo uma adaptação na absorção de Zn proveniente de dietas ricas em fibra e fitato. Entretanto, no estudo realizado com vegetarianos australianos, Ball \& Ackland $^{1}$ não observaram qualquer relação entre a ingestão de fibras e os níveis de Zn séricos.

Neste estudo, embora as razões molares AF:Zn tenham sido inferiores ao valor crítico $15^{3}$, em ambas as refeições, o valor encontrado na refeição vegetariana foi significantemente maior que na dieta onívora, devido ao maior teor de fitato e menor teor de zinco observado na dieta vegetariana em relação à onívora. Estes resultados sugerem que a refeição onívora apresenta maior biodisponibilidade de zinco em relação à vegetariana e, de acordo com a classificação da $\mathrm{WHO}^{25}$, a refeição vegetariana apresentaria uma biodisponibilidade média para Zn, enquanto a onívora, uma alta biodisponibilidade. Levando em 
conta que apenas cerca de $30 \%$ a $35 \%$ do zinco seja absorvido, em uma dieta de média biodisponibilidade, e cerca de $45 \%$ a $55 \%$ sejam absorvidos em uma dieta com alta biodisponibilidade ${ }^{25}$, então a quantidade de zinco suprida nas duas refeições, seria reduzida a, no máximo, 29\%, $21 \%, 15 \%$ e $11 \%$ do valor de referência para mulheres e homens onívoros e mulheres e homens vegetarianos, respectivamente. Considerando que as refeições foram coletadas no horário de almoço, e que devam representar cerca de $30 \%$ das recomendações diárias de nutrientes para os indivíduos, o risco de deficiência de zinco não deve ser descartado na dieta dos universitários que se alimentam diariamente de uma ou outra refeição no RU da Universidade de Brasília, sendo este risco maior para homens vegetarianos e menor para as mulheres onívoras.

A deficiência de zinco em dietas de regiões menos desenvolvidas no Brasil tem sido descrita na literatura ${ }^{27}$. Tal deficiência está associada, principalmente, a déficit de crescimento, hipogonadismo, infertilidade, depressão do sistema imune, redução do paladar e do apetite ${ }^{29}$, e afeta cerca de 2 bilhões de pessoas, predominantemente, crianças e gestantes nos países em desenvolvimento ${ }^{30}$. O atraso no crescimento é uma das intercorrências clínicas mais estudadas, podendo ser parcialmente corrigido com a oferta maior de zinco dietético ${ }^{31}$.

Um estudo populacional, realizado com jovens do sexo masculino, avaliados durante o alistamento militar, na cidade de São Paulo, revelou que, embora a população apresentasse médias de altura inferiores aos jovens dos Estados Unidos da América e Holanda, havia uma tendência de aumento de estatura na população entre as décadas de 50 e 70. A partir desses resultados, os autores previram que, se mantidas aceleradas as taxas de mudança secular observadas entre as décadas de 50 e 70, os jovens paulistas recuperariam os déficits de altura entre uma a três décadas ${ }^{31}$. Porém, um estudo populacional mais recente, realizado na década de 90 , ainda revelou um déficit de altura em 3,0\% entre os jovens mais ricos e 7,2\% entre os mais pobres da Região Sudeste do Brasil, e para a população brasileira, esse valor foi de $11,4 \%^{32}$, contrariando as expectativas projetadas por França Junior et al ${ }^{31}$. Entre as crianças brasileiras, a realidade não é diferente, cerca 10,5\% apresentavam déficit de altura ${ }^{31}$.

Considerando a presença de fitato, proveniente do feijão ou soja, e o reduzido teor de zinco nas duas dietas e, que o cardápio empregado no RU possa representar a dieta predominante do brasileiro, não seria a baixa biodisponibilidade de zinco na dieta nacional responsável pelo remanescente déficit de altura nos jovens brasileiros? A investigação da oferta de zinco na dieta do brasileiro, bem como a avaliação do estado nutricional em relação ao zinco devem ser estimuladas, para que se possa responder a essa pergunta.

Apesar da importância fisiológica do zinco na manutenção de vários processos no organismo humano, pouco se tem feito para combater a deficiência desse mineral no mundo. As políticas de fortificação são voltadas para a deficiência de ferro que, quando fornecido como suplemento ou na forma de alimento fortificado, compete com o pouco zinco dietético disponível ${ }^{29}$. Os alimentos mais ricos em zinco são as carnes, os frutos do mar, raros na dieta da Região Centro-Oeste e no interior do País, e os cereais integrais, sendo que, nestes, cerca de $80 \%$ deste total é perdido no refinamento. A dieta à base de feijão com arroz, predominante no Brasil, é rica em fitato, inibidor da absorção de zinco e pobre em zinco ${ }^{33}$. Dessa forma, a diversificação da dieta, com inclusão de alimentos fontes de zinco, é essencial para aumentar o aporte dietético desse mineral e diminuir a prevalência de sua deficiência no Brasil.

As limitações do presente estudo englobam o número de refeições avaliadas, a escassez das informações fornecidas pelas tabelas de composição de alimentos disponíveis, assim como a avaliação da biodisponibilidade de minerais por meio da razão molar. Devido às limitações das informações a respeito do teor de micronutrientes nas tabelas de composição de alimentos brasileiras, 
foi utilizada uma tabela americana. Como as condições climáticas e do solo podem interferir no teor de micronutrientes dos alimentos, a tabela utilizada pode não refletir a realidade dos alimentos cultivados e consumidos no Brasil. Além disso, a razão molar entre AF:mineral não abrange características individuais, como o estado de saúde e nutricional do indivíduo, e outros fatores dietéticos, como interação entre micronutrientes e a presença de oxalatos, que podem interferir na biodisponibilidade de minerais, aumentando ou diminuindo sua absorção.

\section{O N CLUS Ã O}

Devido ao teor de Ca e Fe nas refeições onívora e vegetariana analisadas, a presença de fitato parece não representar risco de deficiência desses minerais para indivíduos que se alimentam diariamente no Restaurante Universitário da Universidade de Brasília. Entretanto, o baixo teor de Zn, em ambas as refeições, almoço e jantar, juntamente com a presença do fitato, proveniente principalmente do feijão e da soja, podem representar um risco de deficiência de $Z n$, principalmente para homens vegetarianos.

Dada a presença constante do feijão na dieta nacional, o monitoramento dos teores de fitato e de minerais dietéticos deve ser estimulado, em busca do adequado balanço entre eles, visando a garantir o fornecimento adequado de minerais e otimizar os efeitos benéficos do fitato como hipoglicemiante, anticancerígeno e antioxidante e, portanto, a prevenção de doenças crônicas, como câncer, diabetes, acidentes cardiovasculares e doenças associadas ao estresse oxidativo. Em dietas com reduzido teor de minerais, o processamento dos alimentos, sobretudo com fermentação, capaz de reduzir o teor de fitato, deve ser empregado no preparo dos alimentos, visando a aumentar a biodisponibilidade dos minerais.

\section{A GRADECIMENTOS}

Este trabalho teve o apoio financeiro da Fundação de Empreendimentos Científicos e Tecno- lógicos (FINATEC) e do Conselho Nacional de Pesquisa e Desenvolvimento Tecnológico (CNPq).

\section{COLABORADORES}

E.M.A. SIQUEIRA coordenou o planejamento, análise estatística, a discussão dos dados e a redação final do artigo. J.F.R. MENDES colaborou na montagem dos pratos; pesagem, dosagem de fitato, estimativa da composição em minerais e densidade energética. S.F. ARRUDA participou na avaliação da adequação das dietas e discutiu os dados.

\section{REFERÊ NCIAS}

1. Ball MJ, Ackland ML. Zinc intake and status in australian vegetarians. Br J Nutr. 2000; 83(1): 27-33.

2. Leitzmann C. Vegetarian diets: what are the advantages? Elmadfa I, editor. Diet diversification and health promotion. Forum Nutr Basel. 2005; (57):147-56.

3. Wilson AK, Ball MJ. Nutrient intake and iron status of Australian male vegetarians. Eur J Clin Nutr. 1999; 53(3):189-94.

4. Nieman DC. Physical fitness and vegetarian diets: is there a relation? Am J Clin Nutr. 1999; 70(Suppl):570S-5S.

5. Draper A, Lewis J, Malhotra N, Wheeler E. The energy and nutrient intakes of different types of vegetarian: a case for supplements? Br J Nutr. 1993; 69(1):3-19.

6. Freeland-Graves J. Mineral adequacy of vegetarian diets. Am J Clin Nutr. 1988; 48(3Suppl):859-62.

7. Lonnerdal B, Bell JG, Hendrickx AG, Burns RA, Keen $C L$. Effect of phytate removal on zinc absorption from soy formula. Am J Clin Nutr. 1988; 48(5): 1301-6.

8. Pallauf J, Rimbach G. Nutritional significance of phytic acid and phytase. Arch Anim Nutr. 1997; 50(4):301-19.

9. Plaami S. Myoinositol phosphates: analysis, content in foods and effects in nutrition. Lebenson Wiss U Technol. 1997; 30(7):633-47.

10. Richard SE, Thompson LV. Interactions and biological effects of phytic acid. In: Shahidi F, editor. Antinutrients and phytochemicals in food. ACS symposium series, nr 662. Washington (DC): American Chemical Society; 1997. Chapter 17: 294-312. 
11. Saha PR, Weaver CM, Manson AC. Mineral bioavailability in rats from intrinsically labeled whole wheat flour of various phytate levels. J Agric Food Chem. 1994; 42(11):2531-5.

12. Ellis R, Kelsay JL, Reynolds RD, Morris ER, Moser PB, Frazier CW. Phytate: zinc and phytate X calcium: zinc milimolar ratios in self-selected diets of americans, Asian Indians, and Nepalese. J Am Diet Assoc. 1987; 87(8):1043-7.

13. World Health Organization. Trace elements in human nutrition and health. Geneva; 1996.

14. Latta M, Eskin M. A simple and rapid colorimetric method for phytate determination. J Agric Food Chem. 1980; 28(6):1313-5.

15. Pinheiro ABV, Lacerda EMA, Benzecry EH, Gomes MCS, Costa VM. Tabela para avaliação de consumo alimentar em medidas caseiras. Rio de Janeiro: Metha; 1998.

16. United States Department of Agriculture, Food and Nutrition Information Center. Food Composition: Nutrient Data Laboratory [cited 2006 Apr 20]. Available from: http://www.nal.usda.gov/fnic/ foodcomp/search/

17. Zar JH. Biostatistical analysis. New Jersey: Prentice Hall; 1999.

18. Food and Nutritional Board. Institute of Medicine. National Academy of Sciences. Dietary Reference Intakes (DRIs) for calcium, phosphorus, magnesium, vitamin D e fluoride: Recommended Intakes for individuals, 1997 [cited 2006 Apr 20]. Available from: http: //www.nap.edu

19. Food and Nutritional Board. Institute of Medicine. National Academy of Sciences. Dietary Reference Intakes for vitamin A, vitamin K, arsenic, boron, chromium, iodine, iron, manganese, molybdenum, vanadium and zinc: Recommended Intakes for individuals, 2001 [cited 2006 Apr 20]. Available from: http://www.nap.edu

20. Organização Mundial de Saúde. Junta do Conselho de Especialistas da FAO/WHO/ONU. Necessidades de energia e proteína. São Paulo: Roca; 1998. p.147-8.

21. Kelsay JL, Frazier CW, Prather ES, Canary JJ, Clark WM, Powell AS. Impact of variation in the carbohydrate intake on mineral utilization by vegetarians. Am J Clin Nutr. 1988; 48(3 Suppl): 875-9.

22. Weaver CM, Plawecki KL. Dietary calcium: adequacy of a vegetarian diet. Am J Clin Nutr. 1994; 59 (Suppl):1238S-41S.
23. Hunt JR, Roughead ZK. Nonheme-iron absorption, fecal ferritin excretion, and blood indexes of iron status in women consuming controlled lactoovovegetarian diets for 8 wk. Am J Clin Nutr. 1999; 69(5):944-52.

24. Haddad EH, Berk LS, Kettering JD, Hubbad RW, Peters WR. Dietary intake and biochemical, hematologic, and immune status of vegans compared with nonvegetarians. Am J Clin Nutr. 1999; 70(3 Suppl):586S-S93S.

25. Hunt JR, Matthys LA, Johnson LK. Zinc absorption, mineral balance, and blood lipids in women consuming controlled lactoovovegetarian and omnivorous diets for 8 wk. Am J Clin Nutr. 1998, 67(3):421-30.

26. Knudsen E, Sandstrom B, Solgaard P. Zinc, copper and magnesium absorption from a fiber-rich diet. J Trace Elem Med Biol. 1996; 10(2):68-76.

27. Shrimpson R, França TS, Rocha YR, Golden MHN. Estudos sobre o estado nutricional em relação ao zinco na Amazônia. I níveis de zinco no soro e ingestão de zinco em operários de Manaus. Acta Amazônica. 1983; 13(1):773-94.

28. Torrejón CS, Castillo-Durán C, Hertrampf ED, Ruz M. Zinc and iron nutrition in chilean children fed fortified milk provided by the complementary national food program. Nutrition. 2004; 20(2): 177-80.

29. Prasad AS. Zinc deficiency. BMJ. 2003; 326(22): 409-10.

30. Ploysangam A, Falciglia GA, Brehm BJ. Effect of marginal zinc deficiency on human growth and development. J Trop Ped. 1997; 43(4):192-8.

31. França Júnior IF, Silva GR, Monteiro CA. Tendência secular da altura na idade adulta de crianças nascidas na idade de São Paulo entre 1950 e 1976. Rev Saúde Pública. 2000; 34(6 Supl):102-7.

32. Monteiro CA, Conde WL, Popkin BM. Is obesity replacing or adding to undernutrition? Evidence from different social classes in Brazil. Public Health Nutr. 2002; 5(1A):105-12.

33. Oliveira AC, Reis SMPM, Carvalho EM, Pimenta FMV, Rios KR, Paiva KC, et al. Adições crescentes de ácido fítico à dieta não interferiram na digestibilidade da caseína e no ganho de peso em ratos. Rev Nutr. 2003; 16(2):211-7.

Recebido em: 20/10/20005

Versão final reapresentada em: 28/2/2007 Aprovado em: 8/3/2007 
